\title{
Considerações atuais sobre gravidez na adolescência
}

Current considerations on teenage pregnancy

Camila Cristina Manfré ${ }^{1}$, Sara Gomes de Queiróz ${ }^{1}$ Ângelo do Carmo Silva Matthes²

Palavras-chave: Gravidez na Adolescência

Educação em Saúde

Anticoncepção

Adolescência.

Keywords:

Pregnancy in adolescence

Health Education

Contraception

Adolescent

\section{Resumo}

A gravidez na adolescência vem adquirindo proporções significativas. Estima-se que de 20 a $25 \%$ do total de gestantes no Brasil sejam adolescentes, ou seja, em média, há uma adolescente entre cada cinco mulheres grávidas. 0 presente trabalho buscou, por meio de uma análise da literatura atual, levantar os principais aspectos que envolvem a gravidez na adolescência, bem como suas consequências na vida da adolescente, de seu filho e no meio social em que vive. Foi possível concluir que a atuação dos profissionais de saúde, pais e educadores mostra-se fundamental na construção do conhecimento sobre sexualidade dos adolescentes. A aplicação desse conhecimento é uma forma de prevenir a gravidez na adolescência, evitando que o futuro da adolescente e do seu filho seja comprometido.

\section{Abstract}

Teenage pregnancy has acquired significant proportions. It is estimated that 20 to $25 \%$ of all pregnant women in Brazil are teenagers. The present study, through an analysis of the current literature, examines the main aspects involving pregnancy during adolescence, along with its consequences on the life of young mothers, their children and social environment. We conclude that the efforts of health care professionals, parents and educators are crucial to increase awareness of adolescent sexuality. The application of this knowledge is a way to prevent teen pregnancy, thus avoiding the harmful effects on young mothers and their children.

${ }_{1}^{1}$ Graduanda do $5^{\circ}$ ano do Curso de Medicina do Centro Universitário Barão de Mauá - Ribeirão Preto (SP), Brasil.

2 Docente da disciplina de Tocoginecologia do Curso de Medicina do Centro Universitário Barão de Mauá - Ribeirão Preto (SP), Brasil.

Endereço para correspondência: Camila Cristina Manfré, Rua Princesa Isabel, 731 - Vila Tamandaré, CEP: 14085-120 - Ribeirão Preto/SP, Telefones: (16) 3979-6480/9118-1954, E-mail: camilamanfre@yahoo.com.br 


\section{Introdução}

A gravidez na adolescência vem adquirindo proporções significativas. Estima-se que de 20 a $25 \%$ do total de gestantes no Brasil sejam adolescentes, ou seja, em média, há uma adolescente entre cada cinco mulheres grávidas ${ }^{1}$. Dados do Datasus nos últimos dois anos no Brasil mostram que a incidência da gravidez nesta faixa etária conta com cifras que vão de 16,27 a $25,96 \%{ }^{2}$. Em estudo que analisa dados relativos à America Latina, observa-se que entre os $25 \%$ mais pobres da população um de cada três nascimentos origina-se de mãe adolescente, e nas áreas rurais, essa proporção é ainda maior: $40 \%{ }^{3}$.

Existe uma grande preocupação com as consequências que a maternidade precoce pode acarretar à saúde, à educação e ao desenvolvimento econômico e social. Isso se deve ao fato de esta dificultar o desenvolvimento educacional e social da adolescente, assim como a sua capacidade de utilizar todo o seu potencial individual. Como resultado, observa-se uma taxa maior de evasão escolar, desajustes familiares e dificuldade de inserção no mercado de trabalho ${ }^{4,5}$.

Em algumas situações, a gravidez na adolescência pode vir a ser solução para situações conflituosas e não necessariamente um problema em si, refletindo a complexidade desse tema ${ }^{5}$. Isso pode ser percebido quando são analisados os possíveis fatores etiológicos ligados ao incremento das gestações nessa faixa etária, chegando a uma rede multicausal que torna as adolescentes especialmente vulneráveis ${ }^{6}$.

\section{Metodologia}

O método adotado foi de revisão sistemática da literatura, consistindo na busca minuciosa por artigos relacionados ao tema, utilizando-se as seguintes palavras-chave: 'adolescência', 'adolescente', 'gravidez na adolescência', 'aborto', 'anticoncepção', 'reincidência de gravidez na adolescência'. As buscas foram feitas entre os meses de maio e junho de 2009, sendo utilizadas as seguintes bases de dados: Lilacs, Medline, Biblioteca Cochrane, SciELO. Foram encontrados 60 artigos relacionados ao tema, sendo que destes foram selecionados 41. Os artigos excluídos foram aqueles cujo ano de publicação era inferior a 2000, sendo algumas exceções feitas quando o artigo em questão continha assunto relevante ao tema desta revisão.

\section{Fatores que influenciam a adolescente ficar grávida}

\section{Conhecimento sobre anticoncepção}

Análise do conhecimento sobre anticoncepcionais hormonais entre adolescentes que já ficaram grávidas mostrou que aproximadamente $98 \%$ das adolescentes apresentaram baixo conhecimento tanto objetivo quanto percebido. Conhecimento objetivo refere-se àquilo que o indivíduo realmente sabe a respeito, enquanto o conhecimento percebido mostra a relação de confiança da pessoa com o conhecimento que julga ter. Apenas o maior número de gestações foi preditor de elevado conhecimento objetivo para anticoncepcionais orais, o que sugere que a experiência anterior da gestação tenha motivado a busca por mais conhecimento sobre contracepção ou ainda que as informações adquiridas no pré-natal tenham contribuído para a evolução do conhecimento ${ }^{7}$.

Os baixos níveis de conhecimento objetivo e percebido das adolescentes sobre o uso de anticoncepcionais orais revelam a suscetibilidade das jovens ao comportamento sexual de risco. Para reduzir a incidência e reincidência da gravidez na adolescência e suas consequências negativas é necessária uma abordagem interativa com os adolescentes para elevar seu nível de conhecimento acerca desse assunto ${ }^{7}$. Também fica evidente que existem falhas nas políticas de saúde reprodutiva e sexual vigentes no Brasil. O planejamento familiar deve integrar eixos participativos: adolescentes, pais, educadores e profissionais de saúde e a individualização do adolescente quanto a seus valores, crenças e atitudes determinantes de seu comportamento sexual ${ }^{1}$. Além disso, procurar estimular a educação formal e o autoconhecimento das jovens. Contudo, na prática esses objetivos não têm sido alcançados. A adolescente ainda não tem espaço para participação ativa no conhecimento e na escolha de contraceptivos. A participação das escolas nesse aprendizado também não tem funcionado ${ }^{8}$. As técnicas de informação devem priorizar método participativo, relacionamento humano, troca de ideias sobre sexualidade e contracepção, para permitir conhecimento, autonomia e responsabilidade da adolescente diante do planejamento familiar. Também se faz necessário que tanto a escola quanto o serviço de saúde tenham profissionais treinados para acolher os adolescentes com suas dúvidas sobre como desfrutar de sua sexualidade de forma segura, sem discriminação ou julgamentos, com estratégias que façam sentido nos diferentes contextos socioculturais nos quais os jovens experimentam o sexo ${ }^{7,9,10}$.

Entre as adolescentes, as maiores fontes de informações sobre anticoncepção são o pré-natal e a escola, apesar de isso não interferir em suas atitudes ou práticas relativas à contracepção. Isso pode ser devido à má qualidade das informações divulgadas pelo pré-natal ou falta de interação da equipe médica com a adolescente ${ }^{11}$. Estudo realizado com jovens 
de três capitais brasileiras demonstrou que quase $50 \%$ das informações adquiridas por mães adolescentes não provêm dos pais ou da escola, o que mostra o quanto o pré-natal, se feito corretamente, pode contribuir para o conhecimento da adolescente sobre contracepção ${ }^{12}$.

No estudo de Belo ${ }^{13}$ as adolescentes mostraram ter conhecimento adequado sobre os métodos anticoncepcionais e concordaram com seu uso durante o período da adolescência. A religião, a idade e a classe socioeconômica estão relacionadas a maior ou mais adequado conhecimento dos métodos, enquanto a multiparidade a seu maior uso. Cinquenta e quatro por cento das adolescentes nesse estudo usaram algum método contraceptivo na primeira relação sexual, porém muitas adolescentes não estão cientes dos possíveis riscos associados à iniciação sexual precoce ${ }^{13,14}$. Ao serem tomadas medidas para orientação sexual, deve-se levar em conta que durante as primeiras fases do relacionamento entre os adolescentes, intimidade e maturidade ainda não são suficientes para tomar decisões sobre anticoncepção ${ }^{15}$.

Algumas adolescentes conhecem os métodos anticoncepcionais, porém apresentam uma prática inadequada para sua utilização ${ }^{16}$. O grau de escolaridade dos pais das adolescentes pode estar envolvido nesse ponto. De certa forma, isso aponta para as dificuldades ou mesmo para a ineficiência ou inexistência de diálogos familiares acerca da orientação sexual e, obviamente, para as limitações próprias do núcleo familiar ${ }^{13}$.

$\mathrm{Na}$ maioria dos casos, a maternidade na adolescência resulta de uma gravidez não-planejada e pré-conjugal ${ }^{16}$. Isso pode estar relacionado com os achados de Chalem ${ }^{5}$, em que $7 \%$ das adolescentes foram admitidas em uma maternidade para curetagem pós-abortamento e 930 (93\%), para parturição, sendo que $81,2 \%$ não tinham planejado a gestação e $80,1 \%$ eram primigestas ${ }^{5}$. Sobre a existência da contracepção de emergência, ou "pílula do dia seguinte", muitas adolescentes negaram o conhecimento dessa alternativa de anticoncepção. Outras não sabiam como utilizá-la ou ainda não sabiam o que fazer caso se esquecessem de tomar uma pílula da cartela. Essas situações têm elevado potencial de levar a gestações indesejadas, porém evitáveis ${ }^{7}$.

De 123 jovens entre 18 e 24 anos, $73 \%$ já consideraram a possibilidade de aborto, demonstrando uma expressiva presença da ideia desse recurso frente a uma gravidez indesejada, mesmo em contexto de ilegalidade. Entre os 86 jovens que já tinham experiência de gestação, 27 declararam a prática do aborto, sendo 20 rapazes e 7 moças. Esses resultados indicaram diferenças relativas ao gênero quando se fala em aborto induzido, o que pode ser devido ao fato de os rapazes terem maior facilidade em cogitar o aborto, enquanto as moças enfrentam a culpa por realizá-lo e muitas vezes acabam por assumir a gravide $z^{17}$.

\section{Conhecimento sobre anatomia e sexualidade}

Os adolescentes conhecem mais a anatomia dos órgãos genitais $(44,5 \%)$ do que sua fisiologia $(39 \%)^{6}$. Quanto maior a idade, escolaridade e qualidade de vínculo com o parceiro, mais elevado é o conhecimento sobre fisiologia da reprodução, incluindo o reconhecimento do período fértil. Assim, as adolescentes que tinham entre 10 e 14 anos e baixa escolaridade tinham menos conhecimento sobre reprodução do que as mais velhas e do que as que haviam frequentado a escola por mais tempo e, portanto, tiveram o menor percentual de respostas corretas $(23,5 \%)^{6,7}$. Apesar disso, nem sempre o razoável nível de escolaridade e o conhecimento sobre sexualidade conseguem ser traduzidos em sexo protegido e alterações de comportamento ${ }^{5}$.

Como o desenvolvimento psíquico ocorre após o orgânico, a adolescente pode chegar à maturidade reprodutiva passando a apresentar corpo de mulher jovem e fértil, sem ainda ter amadurecimento emocional para administrar o próprio corpo e lidar com a sexualidade ${ }^{18,19}$. Tal fato resulta, muitas vezes, em comportamentos de risco para a contaminação por doenças sexualmente transmissíveis e para a gravidez ${ }^{9}$. Os problemas enfrentados por adolescentes no exercício de sua sexualidade (gravidez, aborto, doenças sexualmente transmissíveis, uso inadequado dos métodos contraceptivos) constituem um quadro preocupante que, com frequência, é descrito na literatura nacional e internacional como tendo entre uma de suas causas a falta de informação. Tal fato pode ser reflexo da falta de uma educação sexual de qualidade, seja pela dificuldade de abordagem dos assuntos relativos ao corpo e à sexualidade no núcleo familiar, seja pela ausência de programas educativos em escolas e serviços de saúde ${ }^{6}$.

\section{Fatores gerais}

A vida sexual iniciada precocemente se relaciona a uma prole maior ${ }^{20}$. Em estudo de corte transversal com mães adolescentes, Paraguassú ${ }^{11}$ constatou que as adolescentes de 10 a 16 anos apresentaram maior número de filhos, em relação às de 17 a 19 anos $^{21}$.

Caputo $^{22}$ constatou que baixa escolaridade paterna, falta de informação sobre sexualidade e fertilização e o uso frequente de drogas ilícitas por familiar residente no domicílio estiveram associados à gravidez na adolescência. Apesar de não terem sido explorados nesse estudo, foram encontrados 
outros aspectos que influenciam a ocorrência de gravidez na adolescência, tais como a gravidez da mãe na adolescência e não ter sido criada pela mãe biológica. $\mathrm{O}$ uso frequente de drogas ilícitas por familiar residente no domicílio é um fator fortemente associado à gravidez na adolescência, independentemente dos demais. A intenção de cursar a faculdade mostra-se como fator de proteção, principalmente na presença de baixa escolaridade materna ${ }^{22}$.

\section{Influências da gravidez sobre a adolescente}

\section{Implicações obstétricas}

Em estudo realizado por Rocha ${ }^{20}$, envolvendo dois grupos etários de adolescentes grávidas (entre 10 e 15 anos e entre 16 e 19 anos), verifica-se que o grupo de adolescentes primíparas na faixa etária mais jovem revela-se de risco significantemente maior para a ocorrência de recém-nascido (RN) de baixo peso. Neste estudo o risco chegou a ser cerca de quatro vezes maior em relação à faixa etária mais velha. Não se verificou diferença significativa na incidência de partos pré-termos entre os grupos estudados ${ }^{20}$.

A gravidez na adolescência está associada a início tardio do pré-natal e número de consultas de pré-natal abaixo do recomendado, além de baixa escolaridade do filho da adolescente, baixo peso ao nascer e maior incidência de desproporção céfalo-pélvica e pré-eclâmpsia ${ }^{23}$.

Entre as complicações da gestação na adolescência citadas por vários autores encontram-se o abortamento, anemia, distócias de parto e hipertensão arterial específica da gravidez $^{20,23,24}$. Dentre estas, Vieira ${ }^{24}$ destaca o abortamento como uma complicação que pode resultar não apenas em consequências físicas como também psicológicas ${ }^{24}$.

\section{Implicações psíquicas}

Moreira $^{10}$ investigou os conflitos vivenciados pelas adolescentes com a descoberta da gravidez. Quando questionadas sobre como estavam vivenciando esse estado na adolescência, muitas jovens viam a gravidez como um acontecimento indesejado e tinham medo de partilhar sua descoberta com a família ou o companheiro. A não-aceitação da gestação estava relacionada à reação dos pais ou responsáveis e ao baixo nível socioeconômico ${ }^{10}$.

Fatores como famílias desestruturadas, conflitos familiares, dificuldades econômicas, abandono escolar e atividades profissionais precárias também influenciam o processo de adaptação à maternidade, uma vez que as famílias concentram seus esforços na sobrevivência, descuidando muitas vezes das funções educativas e de apoio afetivo. A gravidez afeta não só a vida da adolescente como também a de quem a rodeia, uma vez que exige ajustamentos psicológicos individuais e familiares difíceis de serem elaborados e aceitos ${ }^{16}$.

Sintomas de ansiedade e depressão e uso de tabaco em adolescentes primigestas são mais frequentes em comparação com as adolescentes não-grávidas. Esses problemas requerem especial atenção dos serviços de pré-natal a fim de evitar possíveis prejuízos para a saúde das mães e de seus filhos ${ }^{25}$.

A gravidez na adolescência está associada a risco aumentado de ideação suicida durante a gestação e pós-parto, paralelamente a uma grande incidência de depressão e percepção negativa da rede de apoio social ${ }^{26}$. As adolescentes passam por transformações físicas e psicológicas para as quais não estão preparadas e muitas vezes são abandonadas pelo pai do bebê $\hat{~}^{18}$. Também são frequentes os registros de abusos físico e sexual nessa população ${ }^{27}$, o que se associa à presença de ideação suicida ${ }^{28}$, tentativas de suicídio e sintomatologia depressiva crônica no primeiro ano após o parto ${ }^{29}$. Entre as adolescentes com ideação suicida, a maioria é constituída de moças solteiras e que contam com pouco apoio social, independentemente da idade gestacional ${ }^{30}$.

A violência contra as adolescentes é outra questão importante para ser discutida. Violência sexual, emocional e física muitas vezes é praticada por pessoas da estreita relação com as adolescentes, principalmente familiares e parceiros. A violência física destaca-se por ser um fenômeno complexo, de etiologia multicausal e de difícil controle. Uma das principais dificuldades para a prevenção desse tipo de ocorrência é a subnotificação. Tanto as próprias adolescentes quanto suas mães principalmente, em geral, escondem o fato, camuflando com outras respostas os registros corporais deixados pelas agressões. A maioria dos episódios de violência acontece no momento da revelação da gravidez para a família, quando a relação com o pai da adolescente em geral fica dificultada. Nessa ocasião, muitas vezes ocorrem espancamentos e agressões psicológicas, incluindo a indução ao aborto ${ }^{30}$.

Dentre as implicações psíquicas, também é possível destacar a desestruturação pessoal e familiar, a adoção e o abandono do bebề .

\section{Implicação sociofamiliar}

Socialmente, a gravidez representa realização, saúde e maturidade para a mulher e, nos ambientes nos quais há poucas 
possibilidades de atingir esse reconhecimento por outras vias, a maternidade pode ser uma saída para as adolescentes. Frequentemente, a percepção de falta de alternativas educacionais, profissionais e afetivas desejáveis é um fator motivador para a maternidade ${ }^{31,32}$. Esta pode ser vista pela adolescente como alternativa para conseguir a união com o parceiro, na tentativa de adquirir independência da família de origem e efetividade de sua identidade feminina ${ }^{7}$.

O baixo índice de casamentos formais $(7,2 \%)$ e o grande número de uniões consensuais $(60,6 \%)$ em consequência da gravidez demonstram que, nas classes sociais menos favorecidas, existe uma pressão social para que a adolescente e seu parceiro formalizem uma união e passem a conviver sob o mesmo teto, mesmo sem oficializar o casamento ou ter uma independência financeira ${ }^{5}$. Com isso, mãe, bebê e muitas vezes também o companheiro passam a morar com as famílias de origem, tendo-se assim, vários núcleos familiares convivendo num mesmo espaço físico, onde dividem a renda e a organização familiar ${ }^{5}$. Fato que gera impacto econômico sobre as famílias, principalmente nos grupos de baixa renda e mais carentes que, muitas vezes, não se encontram preparados para arcar com essa demanda ${ }^{20}$.

A precocidade das uniões conjugais devido à gravidez pode contribuir para perpetuação de desvantagem social. Ao assumir e limitar-se ao papel de mãe e dona de casa, as adolescentes abandonam os estudos, o que diminui suas possibilidades de escolarização e formação profissional, resultando em prejuízo ao seu potencial produtivo ${ }^{7}$. A frequente baixa escolaridade e a falta de profissionalização entre as gestantes e mães adolescentes dificultam sua inserção no mercado competitivo de trabalho, implicando empregos de baixa remuneração e qualificação ${ }^{10}$. Em consequência, as adolescentes acabam perpetuando a dependência financeira dos pais ou passam a depender do companheiro, além de sofrer com a exposição a riscos sociais como uso de drogas, privações afetivas e culturais ${ }^{7}$.

O perfil desfavorável das mães adolescentes pode ser minimizado pela mudança significativa da situação conjugal para casada ou amasiada ${ }^{33}$. Esse suporte familiar é reconhecido como facilitador de um bom desempenho do crescimento e desenvolvimento infantil. A família é considerada a entidade social básica, e ter uma família bem estruturada constitui fator de proteção para o desenvolvimento e o crescimento da criança. Muitas mães adolescentes continuam morando com seus pais após o parto e contam com a ajuda de seus familiares, o que contribui para minimizar o risco de doenças no primeiro ano de vida de seus filhos ${ }^{32,33}$. A gravidez na adolescência nem sempre é percebida como um problema, especialmente quando há aceitação e apoio familiar ${ }^{18}$. Isso faz com que a adolescente aceite mais facilmente a gravidez e consiga adaptar-se a ela. As adolescentes que permaneceram com o pai do seu filho e puderam contar com o apoio familiar demonstraram ter maior disponibilidade materna, conseguindo estabelecer uma relação mãe-filho adequada ${ }^{16}$.

\section{Implicações para o desenvolvimento da adolescente}

A gravidez precoce e não-planejada pode resultar em sobrecarga psíquica, emocional e social para o desenvolvimento da adolescente, contribuindo para alterações no seu projeto de vida futura, assim como na perpetuação do ciclo de pobreza, educação precária, falta de perspectiva de vida, lazer e emprego e, consequentemente, na busca de melhores condições de vida. A instabilidade econômica contribui para uma reação em cadeia. Primeiro a adolescente abandona os estudos para desempenhar outras responsabilidades decorrentes do novo papel que assume, o de mãe. Em seguida, a baixa escolaridade e grau de instrução precário levam à dificuldade de inserção no mercado de trabalho, bem como causam comprometimento da estabilidade conjugal e do estado de saúde da adolescente e de seu filho, principalmente na ausência do suporte da família ${ }^{21}$.

O projeto de vida da adolescente fica, portanto, extremamente comprometido com a gravidez precoce, tornando distante a possibilidade de obter sucessos que poderiam melhorar suas condições socioeconômicas ${ }^{4}$.

Estudos mostram que a taxa de fecundidade é inversamente proporcional ao nível de escolaridade. No estudo de Chalem $^{5}$ e de Souza ${ }^{7}$, a interrupção dos estudos ocorreu em mais de $60 \%$ dos casos. Esse fato corrobora outros estudos que afirmam que o abandono dos estudos contribui para comprometer a educação e o alcance de melhores postos de trabalho pelas adolescentes, constituindo um meio de manutenção do ciclo de baixa escolaridade e pobreza ${ }^{5,7,21}$. Isso porque uma das poucas opções de inserção social e de ascensão econômica se dá por meio do sistema educacional, e sem o acesso a ele haverá consequências graves para a adolescente e seu filho, bem como para a sociedade em geral ${ }^{5,7}$.

Outro fator pouco estudado e que tem grande impacto no futuro da adolescente é a relação entre amamentação e o risco de câncer de mama. Ao engravidar, as mamas passam por um processo de maturação, o que se considera fator de proteção contra o câncer de mama ${ }^{34,35}$. Há evidências também da proteção da amamentação contra alguns tipos de câncer epitelial do ovário ${ }^{35}$. São necessários, porém, mais estudos para verificar se a gravidez e amamentação ocorridas em idade precoce configuram melhor proteção contra esses tipos de cânceres. 


\section{Implicações no desenvolvimento do filho da adolescente}

Em geral, as crianças filhas de mães adolescentes têm menor necessidade de frequentar creches, sugerindo que possam ficar mais tempo em companhia e cuidados de suas mães ou círculo parental ${ }^{18}$. Isso pode vir a ser um fator de vantagem para o crescimento e desenvolvimento da criança, já que na maioria dos casos esta pode desfrutar da companhia de sua mãe por mais tempo do que aquelas filhas de mães adultas, com a vida familiar e profissional já estruturada. Portanto, as adolescentes precisam receber uma assistência especial, multidisciplinar, para que possam desenvolver a capacidade de cuidar de seu filho de forma suficiente ${ }^{18}$.

As crianças filhas de adolescentes não só apresentam um desenvolvimento semelhante ao dos filhos de adultas, como também o fazem de maneira adequada. Os resultados do estudo podem ter sido favorecidos devido à média de idade ma-

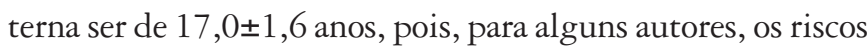
se concentrariam nas idades menores, de 13 a 17 anos $^{33}$.

\section{Papel dos educadores e da sociedade}

Os pais deveriam ser os responsáveis por criar um ambiente propício e acolhedor em seus lares, onde crianças e adolescentes pudessem conversar abertamente sobre questões que estarão diretamente relacionadas ao seu futuro, inclusive a sexualidade, influenciando diretamente em suas escolhas e perspectivas de vida. O bom relacionamento entre pais e filhos constitui um forte alicerce para a formação da personalidade dos futuros adultos ${ }^{4,10}$.

A deficiência na criação desse ambiente propício associada à ausência de serviços de saúde que tenham programas específicos para atender à demanda dos adolescentes faz com que eles procurem obter informações em fontes nem sempre corretas, tais como revistas, rodas de amigos e familiares ${ }^{4,10,24}$. Há uma grande necessidade de que as Unidades Básicas de Saúde tenham profissionais treinados, prontos para orientação, prevenção, acolhimento e esclarecimento de dúvidas dos adolescentes e quando necessário, da adolescente grávida ${ }^{4,10}$.

Como forma de prevenção, são necessários programas que tratem de temas como sexualidade, gravidez, prevenção de doenças sexualmente transmissíveis e síndrome da imunodeficiência adquirida (Aids). Estes devem, sobretudo, considerar os aspectos culturais e socioeconômicos da comunidade em que são desenvolvidos para que os adolescentes consigam transportar as informações recebidas para sua própria realidade. É importante que sejam utilizados recursos didáticos que os sensibilizem, além de que estes devem ser desenvolvidos de acordo com as necessidades apontadas pelos próprios adolescentes, tornando-os ativos no processo de aprendizagem $^{10}$.

A barreira psicossocial é a que mais dificulta a relação dos adolescentes com o sistema de saúde e é representada pela dificuldade deles em dialogar sobre sexualidade com profissionais do sexo oposto. Em geral, as adolescentes grávidas preferem ser atendidas por profissionais do mesmo sexo e esse aspecto deve ser levado em consideração pela equipe de saúde ${ }^{36}$.

Os profissionais envolvidos no processo da maternidade na adolescência têm grande desafio ao lidar com as mudanças e descobertas pelas quais passa a mãe adolescente, que muitas vezes tem dificuldades para entender o significado da maternidade ${ }^{16}$. É necessário que o profissional esteja isento de preconceitos e tenha disponibilidade para permitir a troca de sentimentos e emoções com a adolescente, esclarecendo suas dúvidas e deixando-a expressar seus anseios e preocupações $^{37}$.

As jovens mães devem ser apoiadas em suas representações maternas e na prestação dos cuidados ao bebê, sendo importante o desenvolvimento de programas de intervenção que esclareçam as mães adolescentes ao longo da gravidez e as apoiem em seu processo de adaptação, tentando promover uma boa competência parental ${ }^{16}$. Também é preciso ter em mente que uma adequada orientação para as jovens gestantes pode prevenir a incidência de abortamentos ${ }^{20,24}$, bem como a reincidência da gravidez ${ }^{38}$.

\section{Reincidência de gravidez}

$\mathrm{Na}$ ausência de acompanhamento pós-parto, a reincidência ocorre em torno de $30 \%$ no primeiro ano, $50 \%$ no segundo ano $^{39}$ e até $61 \%$ cinco anos após a primeira gravidez, sendo que cerca de $40 \%$ dessas adolescentes tinha engravidado mais de uma vez nesse período ${ }^{40}$. $\mathrm{O}$ combate à reincidência constitui grande desafio das políticas de planejamento familiar, em especial na adolescência, já que quando não se alcança a inclusão social da adolescente grávida, há maior tendência a recidivas e, muitas vezes, em pior situação que a primeira ${ }^{7,38}$.

A repetição de nova gestação indesejada ainda na adolescência reflete que nem a vivência da gestação nem suas consequências são efetivas para o desenvolvimento de um comportamento sexual responsável, capaz de romper um círculo vicioso 5 . O implemento de políticas de prevenção voltadas para essa faixa etária poderia fazer com que cada vez menos adolescentes participassem dessa estatística. Evitar a 
gravidez e, mais ainda, a repetição desta nesse grupo etário específico fortalecerá as perspectivas de futuro das jovens ${ }^{18}$.

\section{Conclusão}

Por esta revisão, verifica-se que a atenção à saúde da adolescente deve ser mais incisiva por parte dos educadores e do governo, pois a falta de conhecimento sobre anticoncepção, anatomia e sexualidade invariavelmente implica gravidez não-programada. Esta resulta em repercussões indesejáveis para o futuro desses indivíduos, com implicações para sua vida pessoal e social. Existe uma carência de programas específicos para o público adolescente e fica claro que ações de orientação e prevenção implementadas desde a atenção básica, envolvendo todos os profissionais da equipe de saúde, podem ser eficientes no sentido de promover o conhecimento das adolescentes da comunidade, prevenindo a gravidez indesejada na adolescência e suas possíveis repercussões negativas.

\section{Referências}

1. Brasil. Ministério da Saúde. Cadernos da Juventude, Saúde e Desenvolvimento, Brasília (DF): Ministério da Saúde; ago 1999. v-1.

2. Brasil. Ministério da Saúde. DATASUS. Situação de Saúde - Brasil. Brasília; 2010. Disponível em: <http://tabnet.datasus.gov.br/cgi/deftohtm.exe?siab/ cnv/SIABSBR.DEF>. Acesso em: 25 fev. 2010.

3. Kliksberg B. O contexto da juventude na América Latina e no Caribe: as grandes interrogações. Rev. Adm. Pública. 2006;40(5):909-42.

4. Bareiro AOG. Gravidez na adolescência: seus entornos, suas peculiaridades e o ponto de vista da adolescente. Rev Bras Med Fam Com. 2005;1(3):6071.

5. Chalem E, Mitsuhiro SS, Ferri CP, Barros MCM, Guinsburg R, Laranjeira R. Gravidez na adolescência: perfil sócio-demográfico e comportamental de uma população da periferia de São Paulo, Brasil. Cad. Saúde Pública. 2007;23(1):177-86.

6. Carvacho IE, Silva JLP, Mello MB. Conhecimento de adolescentes grávidas sobre anatomia e fisiologia da reprodução. Rev Assoc Med Bras. 2008;54(1):29-35.

7. Sousa MCR, Gomes KRO. Conhecimento objetivo e percebido sobre contraceptivos hormonais orais entre adolescentes com antecedentes gestacionais. Cad. Saúde Pública. 2009;25(3):645-54.

8. Costa AM. Atenção integral à saúde das mulheres: Quo Vadis? Uma avaliação da integralidade na atenção à saúde das mulheres no Brasil [tese]. Brasília: Faculdade de Ciências da Saúde da Universidade de Brasilia; 2004.

9. Gontijo DT, Medeiros M. "Tava morta e revivi": significado de maternidade para adolescentes com experiência de vida nas ruas. Cad. Saúde Pública. 2008;24(2):469-72.

10. Moreira TMM, Viana DS, Queiroz MVO, Jorge MSB. Conflitos vivenciados pelas adolescentes com a descoberta da gravidez. Rev Esc Enferm USP. 2008;42(2):97-107.

11. Costa MPSSM. Conhecimento, atitude e práticas sobre métodos contraceptivos entre adolescentes com antecedentes reprodutivos em Teresina-PI [dissertação]. Teresina: Universidade Federal do Piauí; 2006.

12. Teixeira AMFB, Knauth DR, Fachel JMG, Leal AF. Adolescentes e uso de preservativos: as escolhas dos jovens de três capitais brasileiras na iniciação e na última relação sexual. Cad Saúde Pública. 2006;22(7):1385-96.

13. Belo MAV, Silva JLP. Conhecimento, atitude e prática sobre métodos anticoncepcionais entre adolescentes gestantes. Rev Saúde Pública. 2004;38(4):479-87.
14. Flores CE, Nuñez J. Teenage childbeering in Latin American countries. Washington (DC): Inter-American Development Bank; 2000.

15. Hoga LAK. Adolescent maternity in a low income community: experiences revealed by oral history. Rev Latino-Am Enferm. 2008;16(2):280-6.

16. Carlos Al, Pires A, Cabrita T, Alves H, Araújo C, Bentes MH. Comportamento parental de mães adolescentes. Análise Psicológica. 2007;25(2):183-94.

17. Peres SO, Heilborn ML. Cogitação e prática do aborto entre jovens em contexto de interdição legal: o avesso da gravidez na adolescência. Cad. Saúde Pública. 2006;22(7): 1411-20.

18. Gravidez na adolescência. Folha de São Paulo. 2009 mai 31. Editoriais. p. A2.

19. Berlofi LM, Alkimin ELC, Barbieri M, Guazzelli CAF, Araújo FF. Prevenção da reincidência de gravidez em adolescentes: efeitos de um programa de planejamento. Acta Paul Enferm. 2006;19(2):196-200.

20. Rocha RCL, Souza E, Guazzelli APF, Filho AC, Soares EP, Nogueira ES. Prematuridade e baixo peso entre recém-nascidos de adolescentes primíparas. Rev Bras Ginecol Obstet. 2006;28(9):530-5.

21. Paraguassú ALCB, Costa COM, Sobrinho CLN, Patel BN, Freitas JT, Araújo FPO. Situação sociodemográfica e de saúde reprodutiva pré e pós-gestacional de adolescentes, Feira de Santana, Bahia, Brasil. Ciên Saúde Coletiva. 2005;10(2):373-80.

22. Caputo VG, Bordin IA. Gravidez na adolescência e uso freqüente de álcool e drogas no contexto familiar. Rev Saúde Pública. 2008;42(3):402-10.

23. Santos GHN, Martins MG, Sousa MS. Gravidez na adolescência e fatores associados com baixo peso ao nascer. Rev Bras Ginecol Obstet. 2008;30(5):224-31.

24. Vieira LM, Goldberg TBL, Saes SO, Dória AAB. Abortamento na adolescência: um estudo epidemiológico. Ciên Saúde Coletiva. 2007;12(5):1201-08.

25. Caputo VG, Bordin IA. Problemas de saúde mental entre jovens grávidas e não-grávidas. Rev Saúde Pública. 2007;41(4):573-81.

26. Vasquez R, Piñeros S. Psicopatologia en madres adolescentes. Pediatria (Bogotá). 1997;32(4):229-38.

27. Covington DL, Dalton UK, Diehl SJ, Whight BD, Piner $\mathrm{MH}$. Improving detection of violence among pregnant adolescents. J Adolesc Health. 1997;21(1):18-24.

28. Koniak-Griffin D, Walker DS, Traversay J. Predictors of depression symptoms in pregnant adolescents. J Perinatol. 1996;16(1):69-76.

29. Lesser J. Childhood abuse, depression, and maternal role attainment in adolescent mothers. J Adolesc Health. 1998;22(2):137.

30. Monteiro CFS, Costa NSS, Nascimento PSV, Aguiar YA. A violência intrafamiliar contra adolescentes grávidas. Rev Bras Enferm. 2007;60(4):373-6.

31. Levandowski DC, Piccinini CA, Lopes RCS. Maternidade adolescente. Estud de Psicol. 2008;25(2):251-63.

32. Carvalho GM, Merighi MAB, Jesus MCP. Recorrência da parentalidade na adolescência na perspectiva dos sujeitos envolvidos. Rev Texto Contexto Enferm. 2009;18(1):17-24.

33. Vieira MLF, Bicalho GG, Silva JLCP, Barros Filho AA. Crescimento e desenvolvimento de filhos de mães adolescentes no primeiro ano de vida. Rev Paul Pediatr. 2007;25(4):343-8.

34. Marcus PM, Baird DD, Millikan RC, Moorman PG, Qaqish B, Newman B. Adolescent reproductive events and subsequent breast cancer risk. Am J Public Health. 1999;89(8):1244-7.

35. Rea MF. Os benefícios da amamentação para a saúde da mulher. J Pediatr. 2004;80(5 suppl):S142-S146.

36. Carvacho IE, Silva JLP, Mello MB. Fatores associados ao acesso anterior à gestação a serviços de saúde por adolescentes gestantes. Rev Saúde Pública. 2008;42(5):886-94.

37. Bergamaschi SFF, Praça NS. Vivência da puérpera-adolescente no cuidado do recém-nascido, no domicílio. Rev Esc Enferm USP. 2008;42(3):454-60.

38. Duarte CM, Nascimento VB, Akerman M. Gravidez na adolescência e exclusão social: analise de disparidades intra-urbanas. Rev Panam Salud Publica. 2006;19(4):236-43.

39. Crittenden CP, Boris NW, Rice JC, Taylor CA, Olds CL. The role of mental health factors, behavioral factors, and past experiences in the prediction of rapid repeat pregnancy in adolescence. J Adolesc Health. 2009;44(1):25-32.

40. Bruno ZV, Feitosa FEL, Silveira KP, Morais IQ, Bezerra MF. Reincidência de gravidez em adolescentes. Rev Bras Ginecol Obstet. 2009;31(10):480-4. 